\title{
Generation and Characterization of Novel Human IRAS Monoclonal Antibodies
}

\author{
Bo Wang, ${ }^{1}$ Ying Liu, ${ }^{1}$ Yajun Shan, ${ }^{2}$ Zhenyu Yao, ${ }^{2}$ Xiaolan Liu, ${ }^{2}$ \\ Ruibin Su, ${ }^{1}$ Qihong Sun, ${ }^{2}$ Yuwen Cong, ${ }^{2}$ and Jin $\mathrm{Li}^{1}$ \\ ${ }^{1}$ Department of Pharmacology, Beijing Institute of Pharmacology and Toxicology, Beijing 100850, China \\ ${ }^{2}$ Department of Pathophysiology, Beijing Institute of Radiation Medicine, Beijing 100850, China
}

Correspondence should be addressed to Jin Li, jinli9802@yahoo.com

Received 10 February 2009; Revised 18 May 2009; Accepted 5 June 2009

Recommended by Val J. Watts

Imidazoline receptors were first proposed by Bousquet et al., when they studied antihypertensive effect of clonidine. A strong candidate for I1R, known as imidazoline receptor antisera-selected protein (IRAS), has been cloned from human hippocampus. We reported that IRAS mediated agmatine-induced inhibition of opioid dependence in morphine-dependent cells. To elucidate the functional and structure properties of I1R, we developed the newly monoclonal antibody against the N-terminal hIRAS region including the PX domain (10-120aa) through immunization of BALB/c mice with the NusA-IRAS fusion protein containing an IRAS N-terminal (10-120aa). Stable hybridoma cell lines were established and monoclonal antibodies specifically recognized full-length IRAS proteins in their native state by immunoblotting and immunoprecipitation. Monoclonal antibodies stained in a predominantly punctate cytoplasmic pattern when applied to IRAS-transfected HEK293 cells by indirect immunofluorescence assays and demonstrated excellent reactivity in flow immunocytometry. These monoclonal antibodies will provide powerful reagents for the further investigation of hIRAS protein functions.

Copyright ( $) 2009$ Bo Wang et al. This is an open access article distributed under the Creative Commons Attribution License, which permits unrestricted use, distribution, and reproduction in any medium, provided the original work is properly cited.

\section{Introduction}

Imidazoline receptors were first proposed by Bousquet et al., when they studied antihypertensive effect of clonidine [1]. Based on their physiologic and pharmacological properties, imidazoline receptors are classified into three main types: I1R, I2R, and I3R [2-4]. I1R and I2R have been implicated in hypertension and psychiatric disorder regulation, respectively, while I3R may be involved in insulin secretion [5-9]. Compared with mitochondrial I2R, which resides within the monoamine oxidase protein [10], the clonidine-preferring imidazoline binding sites (known as I1R) are localized to plasma membrane fractions $[11,12]$ and specifically to synaptic plasma membranes [13].

A strong candidate for I1R, known as imidazoline receptor antisera-selected protein (IRAS), has been cloned from human hippocampus [14]. hIRAS is a larger protein of 1504 amino acids consisting of an $\mathrm{NH}_{2}$-terminal phox (PX) domain, 5 putative leucine-rich repeats, a predicted coiledcoil domain, and a long $\mathrm{COOH}-$ terminal region. Several evidence supported the identity of native IIR and IRAS protein in tissue distributions, ligand binding properties, some cellular functions and downstream signal pathways [14-18]. The murine form of IRAS, Nischarin, truncated at the N-terminal 244 amino acids including the PX domain compared with the hIRAS, was a soluble cytosolic protein involved in cytoskeletal organization [19]. It has been shown that decreasing the expression of rat IRAS or Nischarin in PC12 rat pheochromocytoma cells could attenuate the activation of extracellular signal-regulated kinase (ERK) or reduce the radioligand binding to $\mathrm{I} 1 \mathrm{R}$, which further supported that hIRAS or Nischarin might serve as I1R itself, or at least a functional subunit of I1R [20]. Recently, Molderings et al. have reported that the "I1-imidazoline receptors" mediating effects of clonidine and moxonidine in PC12 and the transfected HEK293 cells belonged to the S1P-receptor family, in particular, representing a mixture of sphingosine-1-phosphate (S1P)1- and S1P3-receptors and/or heterodimers of both. It was then proposed that an increased expression of IRAS or Nischarin may improve 
the receptor-trafficking from cytosolic S1P-receptors to the cell membrane and thereby increase the number of binding sites in the plasma membrane for radioligand binding [21]. In our previous study, we also reported that IRAS mediated agmatine-induced inhibition of opioid dependence in morphine-dependent cells [22]. Despite intensive efforts, the molecular base of the I1R had not yet been elucidated.

To elucidate the functional and structure properties of I1R, several different epitope-specific antisera against IRAS have been raised in rabbits [23]. Because of IRAS splice variants or nonspecificity of these antisera, more sizes of IRAS $(\approx 33, \approx 85, \approx 170 \mathrm{KDa})$ have been visualized in various tissue and cells, which limited their uses on western blot. IRAS was reported to target to the endosomes by a combined action of a PX domain and a coiled-coil region. The PX domain, consisting of 10-130 amino acids, was first identified from the sequence analysis of two $\mathrm{SH} 3$ domain-containing cytosolic components of NADPH oxidase, p47phox and p40phox [24]. Therefore, in the present study, we developed the newly monoclonal antibody against the N-terminal hIRAS region including the PX domain (10-120aa). This development has great utility for immunoblotting, indirect immunofluorescent staining, immunoprecipitation, and flow cytometry. These monoclonal antibodies will provide powerful reagents for the further investigation of hIRAS protein functions.

\section{Materials and Methods}

2.1. Generation of the NusA-IRAS(10-120aa) Fusion Protein. E. coli BL21(DE3) (F-ompT gal dcm hsdSB (rB-mB-) $\lambda$ (DE3) (Novagen) cells were transformed with recombinant plasmid, pET43.1-IRAS(10-120aa). Transformants were selected from ampicillin-containing Luria Bertani (LB) lates. Selected colonies were cultured in ampicillincontaining LB media. Isopropyl $-\beta$-D-thio galactopyranoside (IPTG) was added to a final concentration of $1 \mathrm{mM}$. The incubation was continued for 3 hours at $30^{\circ} \mathrm{C}$. Cells were harvested, mixed with lysis buffer (phosphate buffered saline [pH 7.3], $1.0 \mathrm{mM}$ EDTA, $1.0 \mathrm{mM}$ phenylmethylsulfonyl fluoride, $0.5 \mathrm{mg} / \mathrm{mL}$ lysozyme (Roche)), and sonicated. The high-speed supernatant which contained the pET43.1IRAS(10-120aa) soluble protein fraction was loaded onto $10 \mathrm{~mL} \mathrm{Ni}^{2+}$-NTA agarose columns. The fusion protein was eluted with lysis buffer with $400 \mathrm{mM}$ imidazole. Eluted proteins were electrophoresed on SDS-PAGE gels. Expression yields were analyzed with the Quantity One quantitative software (Bio-Rad) based on relative band intensities of comassie blue stains. Purified proteins were detected with western blot using anti-His mAbs (Santa Cruz).

2.2. Antiserum Titer Determination by Indirect Enzymelinked Immunosorbent Analysis (ELISA). The purified NusA$\operatorname{IRAS}_{(10-120 \mathrm{aa})}$, diluted to $10 \mu \mathrm{g} / \mathrm{mL}$ in $50 \mathrm{mM}$ carbonate salt buffer ( $\mathrm{pH}$ 9.6), was coated on plates at $100 \mu \mathrm{L}$ aliquot per well, $4^{\circ} \mathrm{C}$ overnight. The wells were washed three times with PBS-Tween buffer $(0.05 \%$ Tween 20 in PBS). The coated wells were blocked with $200 \mu \mathrm{L}$ of $3 \%$ BSA for 1 hour at $37^{\circ} \mathrm{C}$ and then incubated with $150 \mu \mathrm{L}$ monoclonal antibodies against NusA-IRAS ${ }_{(10-120 a a)}$ fusion protein with different dilutions (from 1 : 1000 to 1 : 25600). After incubation for 1 hour at $37^{\circ} \mathrm{C}$, the wells were incubated with $150 \mu \mathrm{L}$ horseradish peroxidase-conjugated goat antimouse IgG (dilution 1 : 5000, Santa Cruz) for 1 hour at $37^{\circ} \mathrm{C}$ after thorough washing. Peroxidase activity was detected using o-phenylenediamine and $\mathrm{H}_{2} \mathrm{O}_{2}$ as enzyme substrates. Color development was stopped with $2 \mathrm{M}$ of $\mathrm{H}_{2} \mathrm{SO}_{4}$ and the absorbance was measured at $490 \mathrm{~nm}$ using Microplate Reader (Bio-Rad).

\subsection{Production of Antihuman IRAS Monoclonal Antibodies.} The purified NusA-IRAS ${ }_{(10-120 \mathrm{aa})}$ protein $(50 \mu \mathrm{g}$ in a volume of $80 \mu \mathrm{L}$ ) was mixed with an equal volume of Freund's complete adjuvant. The antigen-adjuvant mixture was injected into 6 female $\mathrm{BALB} / \mathrm{c}$ mice and was followed by three booster injections at 2-week interval in incomplete Freund's adjuvant. The mouse with the highest antibody titer tested by ELISA was boosted intraperitoneally with $100 \mu \mathrm{g}$ NusA-IRAS ${ }_{(10-120 \mathrm{aa})}$ protein without adjuvant 3 days before the cell fusion. Feeder layer cells were prepared 1 day prior to fusion. Splenocytes from mice with the highest ELISA antibody titers were fused with murine myeloma cells SP2/0 following standard procedures [25]. Culture supernatants were collected after fusion and initially screened by ELISA with purified NusA-IRAS ${ }_{(10-120 a)}$ fusion proteins as antigens. Positive hybridoma clones were selected with the limiting dilution method, and stable hybridoma clones were obtained after 3 cloning cycles. Isotypes of antibodies were identified with a mouse subisotype panel (Bio-Rad). The pristine-primed BALB/c mice were injected intraperitoneally with $1 \times 10^{6}$ hybridoma cells per mouse in order to acquire abundant mAbs. The ascitic fluid was collected and $\mathrm{mAbs}$ were purified with a protein $\mathrm{A} / \mathrm{G}$ affinity column (Amersham Pharmacia Biotech).

2.4. Western Blotting Analysis. Transfected cells were harvested 48 hours after transfection. Total cell lysate preparation and western blot analysis were performed according to previously described procedures [26]. In brief, cell lysates were prepared, electrophoresed on SDS-PAGE gels, and transferred to polyvinylidene difluoride (PVDF) membranes. Membranes were blocked with $5 \%$ nonfat milk in TBST (20 mM Tris- $\mathrm{HCl}$ [pH 7.5], $150 \mathrm{mM} \mathrm{NaCl}$, and $0.05 \%$ [v/v] Tween 20) and incubated with IRAS or GFP mAbs (Cell Signaling Technology Inc). Blots were incubated with horseradish peroxidase (HRP) conjugated goat antimouse antibodies (Santa Cruz) after primary antibody incubation, and blots were developed with enhanced electrochemiluminescence (ECL, Cell Signaling Technology Inc).

2.5. Confocal Microscopy Analysis. The cellular localization of the IRAS protein was identified according to previously described procedures [27]. Cells on glass cover slips were rinsed with PBS, fixed with 3\% paraformaldehyde for 30 minutes, and permeabilized with $0.2 \%[\mathrm{v} / \mathrm{v}]$ Triton X-100/PBS. Permeabilized cells were incubated with IRAS $\mathrm{mAbs}$ and TRITC-conjugated affinipure goat antimouse 


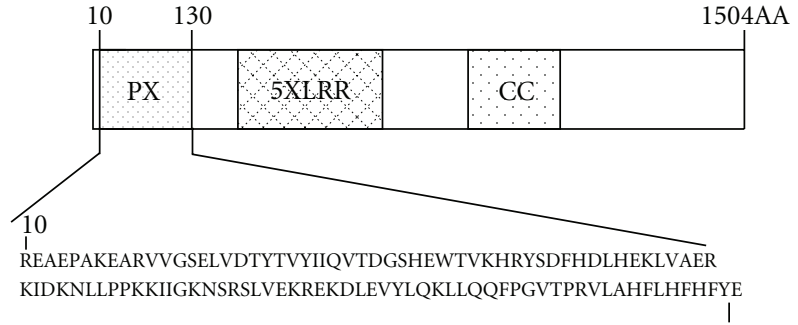

120

(a)

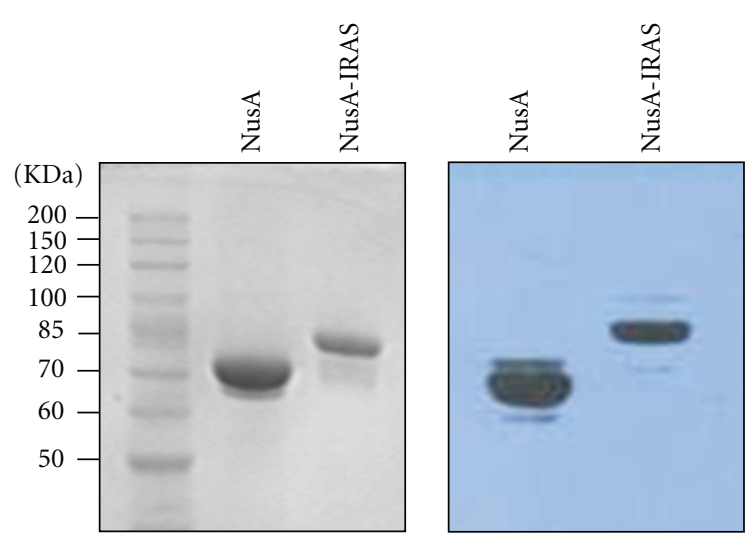

(b)

FIgURE 1: Production of immunogens for human IRAS. (a) Schematic representation of IRAS fusion used for mAb production. The IRAS N-terminal of the PX domain, and the Arg 10 to Glu 120 were expressed as the NusA fusion protein. LRR represents the leucine-rich repeats and CC represents the coiled-coiled region. Numbers represent amino acid positions. (b) Purification and western blot analysis of NusA-IRAS ${ }_{(10-120 a a)}$ fusion proteins. SDSPAGE analysis after filtration through the $\mathrm{Ni}^{2+}$-NTA column (left column) were then immunoblotted with the anti-His mAb (right column). Sizes ( $\mathrm{kDa}$ ) of molecular mass marker were indicated on the left side.

IgG (Santa Cruz) for 1 hour for immunofluorescence assays. Nuclei were stained with $0.1 \mathrm{mg} / \mathrm{mL}$ Hoechst 33342 (Molecular Probes). Specimens were examined by confocal fluorescent microscopy on a Bio-Rad Radiance 2100 System.

2.6. Immunoprecipitation Analysis. The HEK293 cells were transfected with PCMV-myc-IRAS or PCMV-myc plasmid to analyze IRAS mAbs immunoprecipitated with IRAS. Cell lysates were prepared in a modified RIPA buffer (50 mM Tris$\mathrm{HCl}$ [pH 7.5], $150 \mathrm{mM} \mathrm{NaCl}, 1 \%$ [v/v] Nonidet P40, 0.5\% [w/v] sodium deoxycholate) containing Complete Protease inhibitor Cocktail Tablets (Roche). Cell lysates were centrifuged to remove debris. Recovered lysates were incubated with IRAS or c-myc mAbs (Santa Cruz) overnight at $4^{\circ} \mathrm{C}$ followed by protein A/G agarose beads ( $50 \mu \mathrm{L}$, Santa Cruz) for another 3 hours to capture the immunoprecipitates. Recovered immune complexes were extensively washed in lysis buffer. Samples were boiled to release the bound proteins and appropriate supernatant aliquots were run on SDS-PAGE gels for western blot analysis as described above.
2.7. Flow Cytometry. Cells $\left(2 \times 10^{6}\right)$ were transfected with PCMV-myc-IRAS for each sample and fixed with 70\% [v/v] cold ethanol at $4^{\circ} \mathrm{C}$ overnight. Cells were permeabilized in $0.1 \%[\mathrm{v} / \mathrm{v}]$ Triton X-100/PBS and were incubated with IRAS or c-myc mAbs (Santa Cruz). Cells were washed and resuspended in an FITC-conjugated affinipure goat antimouse IgG (Santa Cruz). Finally, cells were washed twice and samples were analyzed on a flow immunocytometry machine (Becton-Dickinson) [28].

2.8. Cell Culture and Transfection. HEK293 cells were grown in Dulbecco's modified Eagle medium (DMEM) (Gibco BRL) supplemented with $10 \%$ fetal bovine serum. Cells were transfected with different plasmid DNAs using a Lipofectamine 2000 (Invitrogen) when cell densities reached 70\% confluence per the manufacturer's instructions. Hybridoma cells were grown in DMEM with 10\% fetal bovine serum.

2.9. Construction of Expression Vector pET43.1a-IRAS, pCMV-myc-IRAS, and pEGFPC1-IRAS. The cDNA fragment of the human IRAS N-terminal (10-120aa) was inserted into the pET-43.1a(+) expression vector (Novagen) by PCR amplification of the human IRAS cDNA (AF082516) using the following oligomers: sense, 5'-CGGGATCCATGGAGCGGGAAGCCGAGCCG-3', and antisense, 5' CGGAATTCATAGAAGTGAAAATGCAAGAAGTG-3'. The full-length human IRAS was obtained by PCR amplification of the entire coding region, and the resulting 4512-bp PCR product was ligated into the pEGPC1 and PCMV-myc vectors in a similar fashion using the following oligomers, respectively: sense, 5' -CGCGAATTCTATGGCGACCGCGCGCACCTTCG-3' , and antisense, $5^{\prime}$-CGGGATCCTAGCCGGTGAGCTCGACAGGC-3', sense, 5' -CGCGAATTCGGATGGCGACCGCGCGCACCTTC-3' ${ }^{\prime}$, and antisense, 5' CCGCTCGAGCTAGCCGGTGAGCTCGACAGGC-3' . All plasmid sequences were confirmed by sequencing analysis.

\section{Results and Discussion}

3.1. Generation of Murine Monoclonal Antibodies (mAbs) against a Recombinant NusA-IRAS (10-120aa) $_{1}$ Fusion Protein. IRAS is a large protein comprising of 1504 amino acids. Its $\mathrm{NH}_{2}$-terminal phox domain is important for membrane association and cellular localization. The N-terminal of IRAS (10-120aa) covering the PX domain was cloned into the pET-43.1a(+) (Figure 1(a)) [29]. The supernatant fusion protein was purified by His-tag affinity purification and was subsequently used to generate the monoclonal antibody. The dissolved protein yielded one major band of $78 \mathrm{kDa}$ expected molecular weight (Figure 1(b)) with high purity and integrity. The NusA protein used as a control generated the $66 \mathrm{kDa}$ expected molecular weight. The recombinant protein was also confirmed with western blot using anti-His mAbs (right column, Figure $1(\mathrm{~b}))$. BALB/c mice $(n=3)$ were

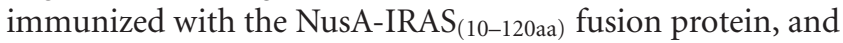
blood was collected from the mice after multiple injections. Antibody titers were tested by ELISA on plates coated with the NusA-IRAS ${ }_{(10-120 a a)}$ protein (data not shown). The 2 mice 

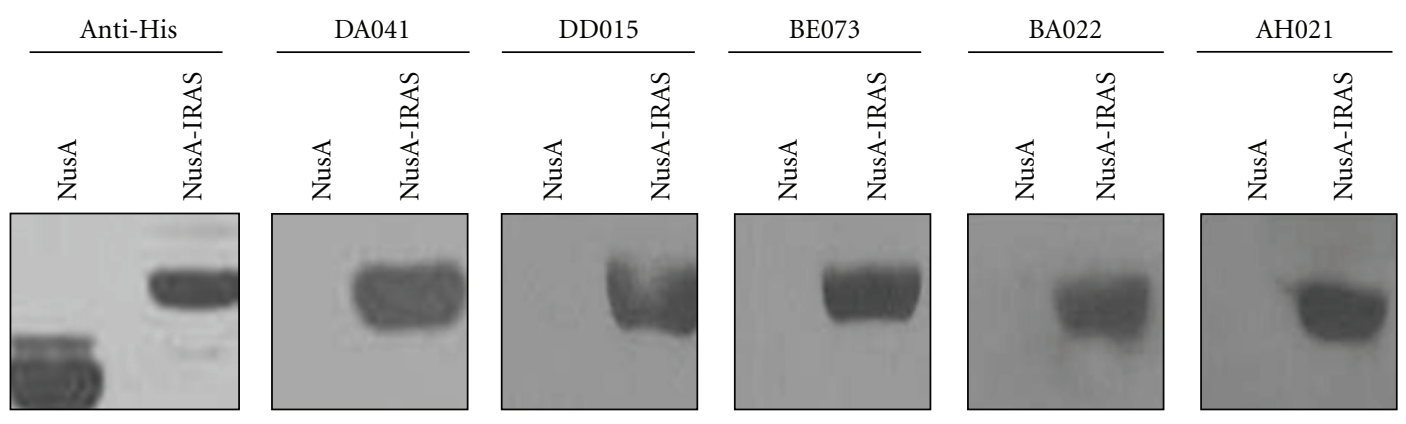

(a)
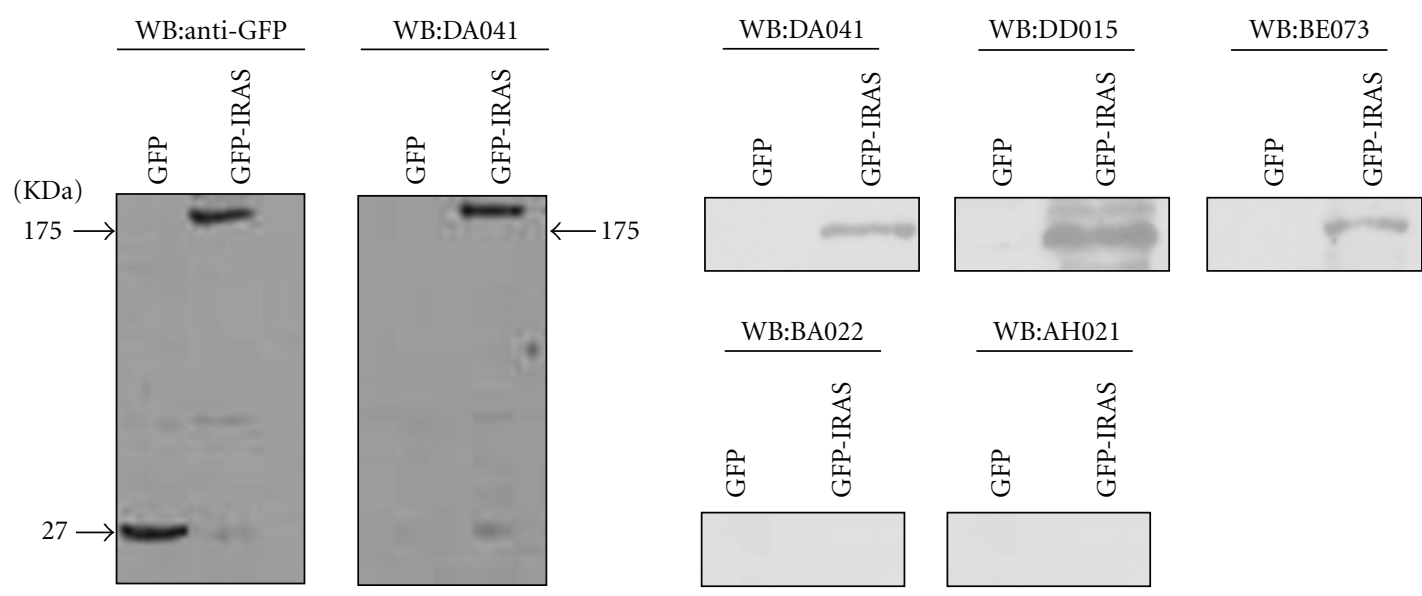

(b)

FIGURE 2: Verification of the specificity of the IRAS mAbs. (a) Identification by western blot using anti-His mAb or the mAbs DA041, DD015, BE073, BA022, and AH021 in NusA and NusA-IRAS ${ }_{(10-120 a a)}$ recombinant proteins. (b) The whole cell extracts derived from HEK293 cells were transfected with pEGFPC1-IRAS and the control vector pEGFPC1 plasmid, respectively. Cell lysates were prepared 48 hours after transfection and were separated by SDS-PAGE. Proteins transferred to PVDF membranes were probed with anti-GFP antibody or the mAbs DA041, DD015, BE073, BA022, and AH021. Sizes (kDa) of molecular mass marker were indicated on the left side.

with the highest titers were sacrificed and spleens from both mice were fused to myeloma cells following standard procedures. Individual hybridomas was grown and 125 hybridomas were further characterized. Supernatant from the growing hybridoma clones was screened with ELISA. Screening was performed on plates coated with NusAIRAS $_{(10-120 a a)}$, NusA protein, and GST-IRAS ${ }_{(10-120 a a)}$ fusion protein to determine antibody specificity. A total of 5 hybridomas (DA041, DD015, BE073, BA022, and AH021) reacted selectively with the NusA-IRAS ${ }_{(10-120 a a)}$ protein in all 3 assays and were further evaluated. Isotype analysis revealed that all mAbs were of the IgG1 subtype.

The immunoreactivities of the 5 representative mAbs with NusA-IRAS ${ }_{(10-120 a a)}$ were shown in Figure 2, all of which specifically recognized a $78 \mathrm{kDa}$ protein band which corresponded to the purified NusA-IRAS ${ }_{(10-120 a a)}$ protein, but not to the $66 \mathrm{kDa}$ NusA protein. Anti-His mAbs reacted with both proteins in the same experiment (Figure 2(a)) as controls. We evaluated the specificity of mAbs in mammalian cells by inserting IRAS cDNA into expression vectors to allow the production of GFP fusion proteins under the control of a CMV promoter. The pEGFPC1 and pEGFPC1-IRAS plasmids were separately transfected into HEK293 cells. Western blot analysis with the anti-GFP antibody demonstrated that chimeric proteins were expressed and migrated separately at expected molecular masses of approximately 19 or $27 \mathrm{KDa}$ (Figure 2(b)). However, the expected $190 \mathrm{kDa}$ band whose molecular weight corresponded to the full-length IRAS protein was only detected with the mAbs DA041, DD015, and BE073 in GFP-IRAS expressed cells. The remaining BA022 and AH021 mAbs were negative (Figure 2(b)). The same samples were also incubated with preimmune serum with no reactivity (data not shown). Results revealed that all 5 selected mAbs specifically recognized bacterially expressed NusA-IRAS ${ }_{(10-120 a a)}$ proteins, but only $3 \mathrm{mAbs}$ recognized recombinant IRAS in mammalian cells.

3.2. Cellular Localization and Flow Cytometry Analysis for IRAS Expression. IRAS mAbs were used to detect the cellular localization of IRAS protein based on specificity characterized by western blot analysis. The pEGFPC1-IRAS plasmid was transfected into HEK293 cells. IRAS protein localization was tested by fluorescence confocal microscopy after 48 hours transfection. GFP-IRAS was primarily located in the cytoplasm in a punctate pattern (Figure 3(a)) with concentration onto discrete loci and spot fluorescence (panel A, E, I, 


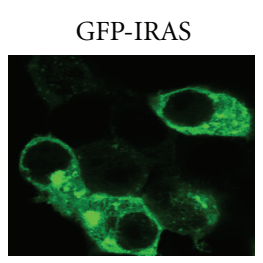

(A)

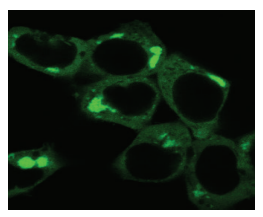

(E)

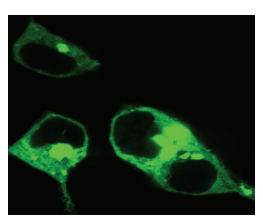

(I)

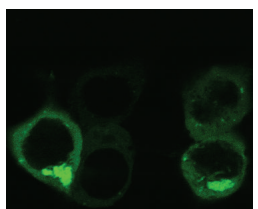

(M)

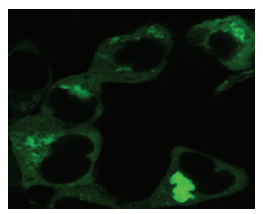

(Q)

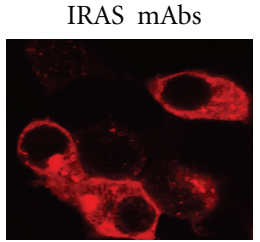

(B)

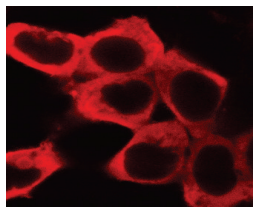

(F)

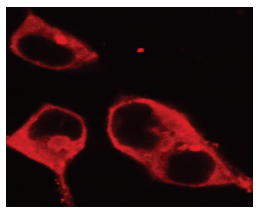

(J)

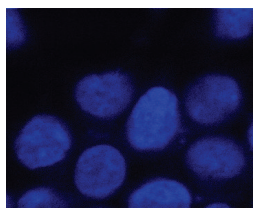

$(\mathrm{N})$

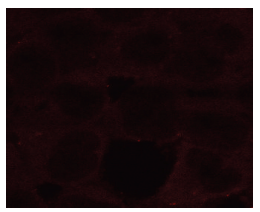

(R)

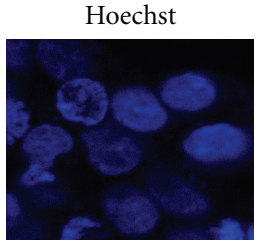

(C)

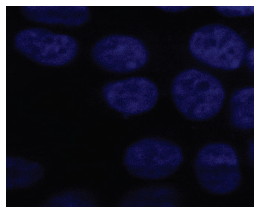

(G)

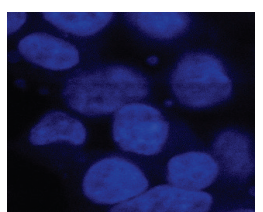

$(\mathrm{K})$

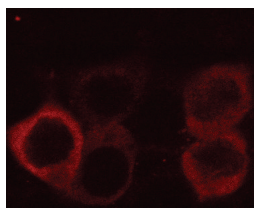

(O)

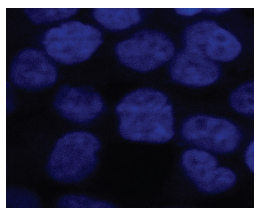

(S)

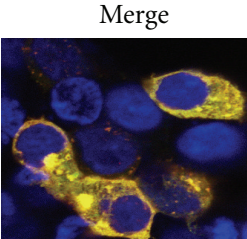

(D)

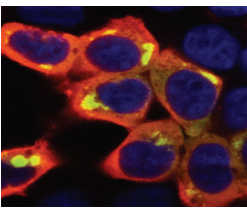

$(\mathrm{H})$

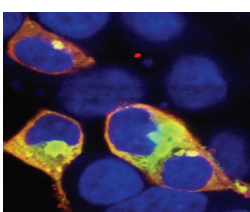

(L)

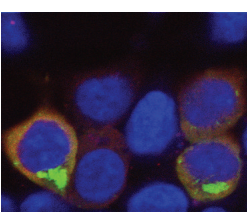

(P)

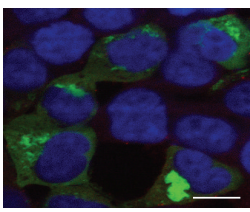

(T)

(a)
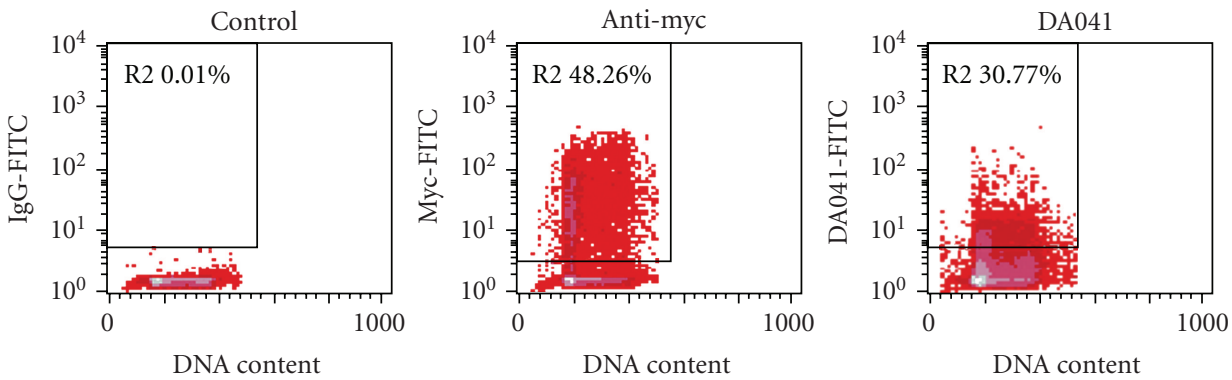

(b)

Figure 3: Binding of IRAS mAbs to IRAS-expressing cells by immunofluorescence and flow cytometry analysis. (a) HEK293 cells were transfected with the plasmid pEGFPC1-IRAS. Cellular localization of IRAS was observed by scanning fluorescence confocal microscopy. Green represented EGFP fluorescence (A, E, I, M, and Q) from the GFP-IRAS fusion protein, blue (C, G, K, O, and S) fluorescence represented Hoechst stained cell nuclei, red (B, F, J, N, and R) fluorescence represented cells expressing GFP-IRAS labeled with TRITC-conjugated goat antimouse antibody using IRAS mAbs as the primary antibody, and yellow (D, H, L, P, and T) represented overlapping green and red fluorescence. All 3 panels had the same field of view. Scale bar, $100 \mu \mathrm{m}$. (b) Samples were collected and separately analyzed by flow cytometry for the ability to bind the preimmune serum (control), the c-myc mAb, and the mAb DA041 after 48 hours transfection of PCMV-myc-IRAS. Results were expressed as histograms with the DNA content on $x$-axis and the number of fluorescent cells on $y$-axis. Cells were distributed in 2 populations, and the second population (R2) with high fluorescence reflected the population of transfected cells recognized by the mAb DA041. 


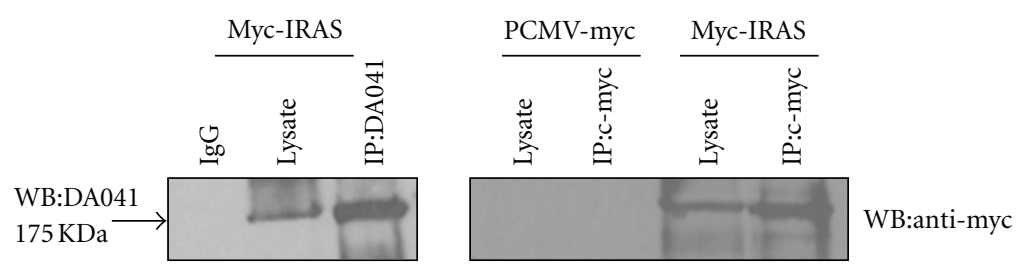

(a)

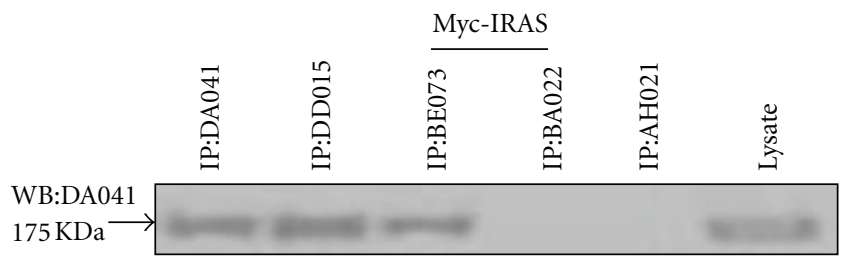

(b)

FIGURE 4: Analysis of immunoprecipitates revealed that the IRAS mAbs specifically recognized the native state of the IRAS protein. The HEK293 cells were transiently transfected with the empty vector PCMV-myc (negative control) and PCMV-myc-IRAS. Whole cell lysates were prepared and immunoprecipitated with the c-myc mAb or the mAbs DA041, DD015, BE073, BA022, and AH021. Immunoprecipitation of the same lysates using mouse normal IgG did not result in the detection of any protein species. Immunocomplexes were analyzed by western blot with indicated antibodies.

$\mathrm{M}$, and Q), as confirmed in previous studies [30]. The mAbs DA041, DD015, and BE073 were used as probes to detect a predominantly punctate cytosolic distribution as expected (panel B, F, G). However, the mAb BA022 homogenously detected the fluorescence distribution over the cytoplasm (panel N) and the mAb AH021 scored negatively (panel $\mathrm{R})$. Merged images revealed that the GFP-IRAS fluorescence significantly coincided with the immunostaining with $\mathrm{mAbs}$ DA041, DD015, and BE073 (panel D, H, and L), but was not reflected by the mAbs BA022 and AH021 (panel P and $\mathrm{T}$ ). Further, the punctate fluorescence patterns appeared to be unique to the mAbs DA041, DD015, and BE073. In addition, we also detected the endogenous IRAS protein with monoclonal antibody DA041 in PC12 cells, showing a strong signal associated with the membrane and faint signal in the cytoplasm (data not shown). These results suggest that the 3 mAbs specifically detect IRAS proteins by immunofluorescence, similar to immunoblotting results.

Flow cytometry is a rapid, quantitative, multiparameter cellular analysis based on the measurement of visible and fluorescent light emission. We chose the representative $\mathrm{mAb}$ DA041 for further characterization based on the strong signals detected by immunoblotting and immunofluorescence. The IRAS CDNA was cloned into the mammalian expression vector PCMV-myc and was expressed in HEK293 cells. The $\mathrm{mAb}$ DA041 and the c-myc mAb (used as a positive control) resulted in significant increases in fluorescence intensity when compared to cells incubated with preimmune serum (Figure 3(b)). The binding efficiencies were $30.77 \%$ by the $\mathrm{mAb}$ DA041 and $48.26 \%$ by the c-myc mAb. No reactivities were detectable in preimmune sera $(0.01 \%)$. These results indicate mAb DA041 is effective for detecting IRAS protein by flow cytometry.

3.3. MAbs IRAS Immunoprecipitates IRAS from Cell Lysates. We next characterized IRAS mAb ability to recognize IRAS proteins in their native states by immunoprecipitation. Cell extracts were immunoprecipitated with the mAb DA041 and analyzed with mAb DA041 immunoblotting after 48-hour transfection with PCMV-myc-IRAS. IRAS was selectively immunoprecipitated from cell lysates that expressed the mycIRAS protein (approximately $175 \mathrm{kDa}$ ), and those which corresponded to the predicted size of the full-length IRAS (Figure 4). Immunoprecipitation of lysates with mouse normal IgG did not result in detection of protein species. Lysates were immunoprecipitated with the c-myc mAb to confirm $\mathrm{mAb}$ specificity, and similarly sized bands were detected. No corresponding band was visualized when the negative control c-myc mAb was used to immunoprecipitate cell lysate of the transfectant expressing the empty PCMV-myc vector. Similar results were obtained with the mAbs DD015 and $\mathrm{BE} 073$ under the same conditions, and the remaining mAbs BA022 and AH021 scored negatively. Therefore, the 3 isolated antibodies specifically recognized native full-length IRAS protein products.

Our results suggested that the mAbs DA041, DD015, and BE073 were reactive for both immunofluorescence and immunoblotting. Therefore, these mAbs likely recognized linear epitopes in the IRAS protein. In addition, the $3 \mathrm{mAbs}$ were capable of recognition of native full-length IRAS proteins by immunoprecipitation. However, the mAbs BA022 and AH021 specifically recognized bacterially expressed IRAS immunogens and did not detect recombinant IRAS in mammalian cells. This could be related to the different IRAS folding patterns, since misfolded IRAS proteins could result in the exposure of unique immunogenic epitopes different from native proteins. The mAbs BA022 and AH021 could be used as backup reagents to safeguard against antibodyspecific artifacts. In addition, analyzing the overall homology of the amino acid sequence of the PX domain, Sorting Nexins 13 (SNX13) is the most relative protein to IRAS [31]. We 
found monoclonal antibody DA041 against hIRAS could not react with SNX13 by immunofluorescence assay (data not shown). It was proposed that these antibodies developed here were specific to the PX domain of IRAS.

In summary, we generated specific mAbs directed against the human IRAS N-terminal. The mAb DA041 exhibited the best performance in a variety of assays including immunoblotting, immunoprecipitation, indirect immunofluorescence stainning, and flow cytometry. Specific mAbs may provide an ideal reagent for further investigation of the function of IRAS proteins.

\section{Acknowledgments}

The authors would like to thank J. E. Piletz for kindly providing the IRAS-pcDNA3.1 plasmid and J. Q. Zheng for advice of English usages throughout the article and Zhiyi Zhang for the assay of confocal microscopy. This work was supported by the National Basic Research Program of China (no. 2003CB515400, no. 2007CB512307, and no. 2009CB202000), National Natural Science Foundation of China 30701016, Beijing Municipal Natural Science Foundation 7082073 .

\section{References}

[1] P. Bousquet, J. Feldman, and J. Schwartz, "Central cardiovascular effects of alpha adrenergic drugs: differences between catecholamines and imidazolines," Journal of Pharmacology and Experimental Therapeutics, vol. 230, no. 1, pp. 232-236, 1984.

[2] P. Ernsberger, M. E. Graves, L. M. Graff, et al., "I1-imidazoline receptors: definition, characterization, distribution, and transmembrane signaling," Annals of the New York Academy of Sciences, vol. 763, pp. 22-42, 1995.

[3] F. Tesson and A. Parini, "Identification of an imidazolineguanidinium receptive site in mitochondria from rabbit cerebral cortex," European Journal of Pharmacology, vol. 208, no. 1, pp. 81-83, 1991.

[4] S. L. F. Chan, C. A. Brown, K. E. Scarpello, and N. G. Morgan, "The imidazoline site involved in control of insulin secretion: characteristics that distinguish it from I1- and I2-sites," British Journal of Pharmacology, vol. 112, no. 4, pp. 1065-1070, 1994.

[5] G. J. Molderings, "Imidazoline receptors: basic knowledge, recent advances and future prospects for therapy and diagnosis," Drugs of the Future, vol. 22, no. 7, pp. 757-772, 1997.

[6] P. Ernsberger, T. H. Damon, L. M. Graff, S. G. Schäfer, and M. O. Christen, "Moxonidine, a centrally acting antihypertensive agent, is a selective ligand for I1-imidazoline sites," Journal of Pharmacology and Experimental Therapeutics, vol. 264, no. 1, pp. 172-182, 1993.

[7] F. Tesson, I. Limon-Boulez, P. Urban, et al., "Localization of I2imidazoline binding sites on monoamine oxidases," Journal of Biological Chemistry, vol. 270, no. 17, pp. 9856-9861, 1995.

[8] R. Raddatz, A. Parini, and S. M. Lanier, "Localization of the imidazoline binding domain on monoamine oxidase B," Molecular Pharmacology, vol. 52, no. 4, pp. 549-553, 1997.

[9] N. G. Morgan, S. L. F. Chan, C. A. Brown, and E. Tsoli, "Characterization of the imidazoline binding site involved in regulation of insulin secretion," Annals of the New York Academy of Sciences, vol. 763, pp. 361-373, 1995.
[10] R. Raddatz, A. Parini, and S. M. Lanier, "Imidazoline/guanidinium binding domains on monoamine oxidases: relationship to subtypes of imidazoline-binding proteins and tissue-specific interaction of imidazoline ligands with monoamine oxidase B," Journal of Biological Chemistry, vol. 270, no. 46, pp. 27961-27968, 1995.

[11] J. E. Piletz, A. C. Andorn, J. R. Unnerstall, and A. Halaris, "Binding of [3H]-p-aminoclonidine to $\alpha 2$-adrenoceptor states plus a non-adrenergic site on human platelet plasma membranes," Biochemical Pharmacology, vol. 42, no. 3, pp. 569-584, 1991.

[12] P. Ernsberger and I.-H. Shen, "Membrane localization and guanine nucleotide sensitivity of medullary I1-imidazoline binding sites," Neurochemistry International, vol. 30, no. 1, pp. 17-23, 1997.

[13] F. M. J. Heemskerk, M. Dontenwill, H. Greney, C. Vonthron, and P. Bousquet, "Evidence for the existence of imidazolinespecific binding sites in synaptosomal plasma membranes of the bovine brainstem," Journal of Neurochemistry, vol. 71, no. 5, pp. 2193-2202, 1998.

[14] J. E. Piletz, T. R. Ivanov, J. D. Sharp, et al., "Imidazoline receptor antisera-selected (IRAS) cDNA: cloning and characterization," DNA and Cell Biology, vol. 19, no. 6, pp. 319-329, 2000.

[15] M. Dontenwill, G. Pascal, J. E. Piletz, et al., "IRAS, the human homologue of Nischarin, prolongs survival of transfected PC12 cells," Cell Death and Differentiation, vol. 10, no. 8, pp. 933-935, 2003.

[16] M. Dontenwill, J. E. Piletz, M. Chen, et al., "IRAS is an antiapoptotic protein," Annals of the New York Academy of Sciences, vol. 1009, pp. 400-412, 2003.

[17] L. Dupuy, D. Urosevic, H. Greney, et al., "I1 imidazoline receptor-mediated effects on apoptotic processes in PC12 cells," Cell Death and Differentiation, vol. 11, no. 9, pp. 10491052, 2004.

[18] J. E. Piletz, G. Wang, and H. Zhu, "Cell signaling by imidazoline-1 receptor candidate, IRAS, and the nischarin homologue," Annals of the New York Academy of Sciences, vol. 1009, pp. 392-399, 2003.

[19] S. K. Alahari, J. W. Lee, and R. L. Juliano, "Nischarin, a novel protein that interacts with the integrin $\alpha 5$ subunit and inhibits cell migration," Journal of Cell Biology, vol. 151, no. 6, pp. 1141-1154, 2000.

[20] Z. Sun, C. H. Chang, and P. Ernsberger, "Identification of IRAS/Nischarin as an I1-imidazoline receptor in PC12 rat pheochromocytoma cells," Journal of Neurochemistry, vol. 101, pp. 99-108, 2007.

[21] G. J. Molderings, H. Bőnisch, M. Brúss, C. Wolf, I. von Kúgelgen, and M. Gőthert, "S1P-receptors in PC12 and transfected HEK293 cells: molecular targets of hypotensive imidazoline I1 receptor ligands," Neurochemistry International, vol. 51, no. 8, pp. 476-485, 2007.

[22] N. Wu, R.-B. Su, B. Xu, et al., "IRAS, a candidate for I1imidazoline receptor, mediates inhibitory effect of agmatine on cellular morphine dependence," Biochemical Pharmacology, vol. 70, no. 7, pp. 1079-1087, 2005.

[23] H. Zhu, J. Hayes, M. Chen, J. Baldwin, and J. E. Piletz, "Relationship between platelet imidazoline receptor-binding peptides and candidate imidazoline-1 receptor, IRAS," Annals of the New York Academy of Sciences, vol. 1009, pp. 439-446, 2003.

[24] C. P. Ponting, "Novel domains in NADPH oxidase subunits, sorting nexins, and PtdIns 3- kinases: binding partners of SH3 domains?" Protein Science, vol. 5, no. 11, pp. 2353-2357, 1996. 
[25] P. N. Nelson, G. M. Reynolds, E. E. Waldron, E. Ward, K. Giannopoulos, and P. G. Murray, "Monoclonal antibodies," Molecular Pathology, vol. 53, no. 3, pp. 111-117, 2000.

[26] X. Yuan, Y. Cong, J. Hao, et al., "Regulation of LIP level and ROS formation through interaction of $\mathrm{H}$-ferritin with G-CSF receptor," Journal of Molecular Biology, vol. 339, no. 1, pp. 131144, 2004.

[27] X. Yuan, J. Li, Y. Shan, et al., "Subcellular localization and membrane association of SARS-CoV 3a protein," Virus Research, vol. 109, no. 2, pp. 191-202, 2005.

[28] L.-W. Deng, I. Chiu, and J. L. Strominger, "MLL5 protein forms intranuclear foci, and overexpression inhibits cell cycle progression," Proceedings of the National Academy of Sciences of the United States of America, vol. 101, no. 3, pp. 757-762, 2004.

[29] R. G. Harrison, "Expression of soluble heterologous proteins via fusion with NusA protein," Innovations, vol. 11, pp. 4-7, 2000.

[30] K.-P. Lim and W. Hong, "Human nischarin/imidazoline receptor antisera-selected protein is targeted to the endosomes by a combined action of a PX domain and a coiled-coil region," Journal of Biological Chemistry, vol. 279, no. 52, pp. 54770-54782, 2004.

[31] B. Zheng, Y. C. Ma, R. S. Ostrom, et al., "RGS-PX1, a GAP for GalphaS and sorting nexin in vesicular trafficking," Science, vol. 294, pp. 1845-1847, 2001. 

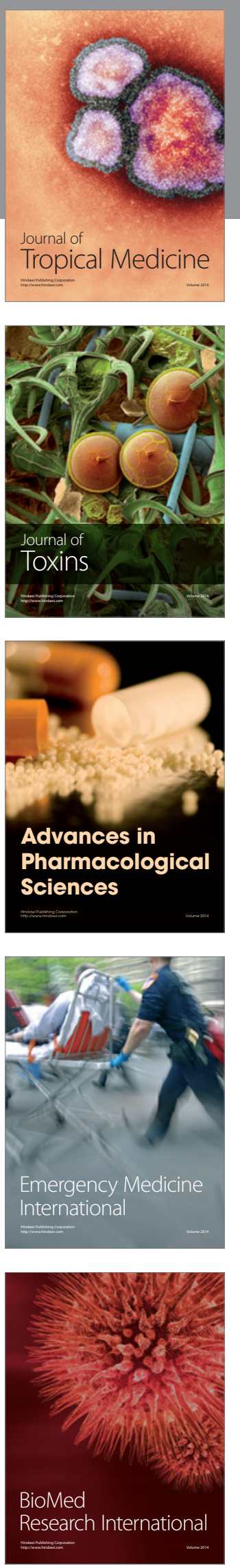
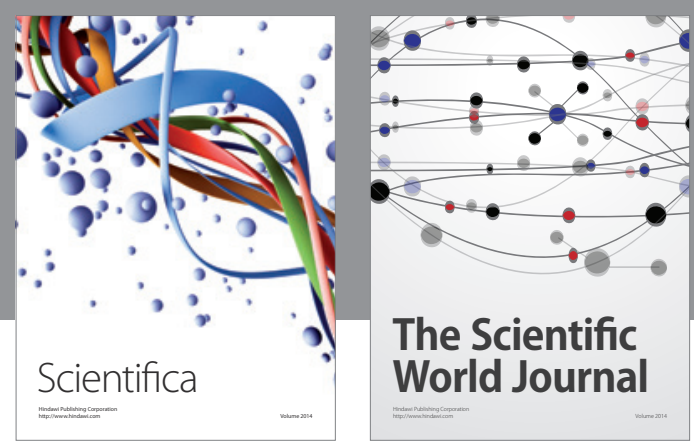

The Scientific World Journal
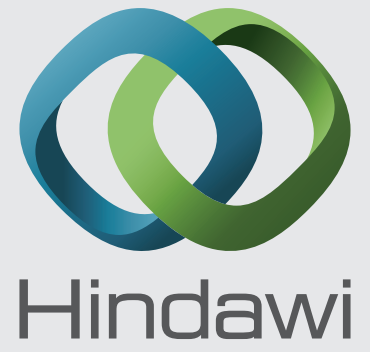

Submit your manuscripts at

http://www.hindawi.com
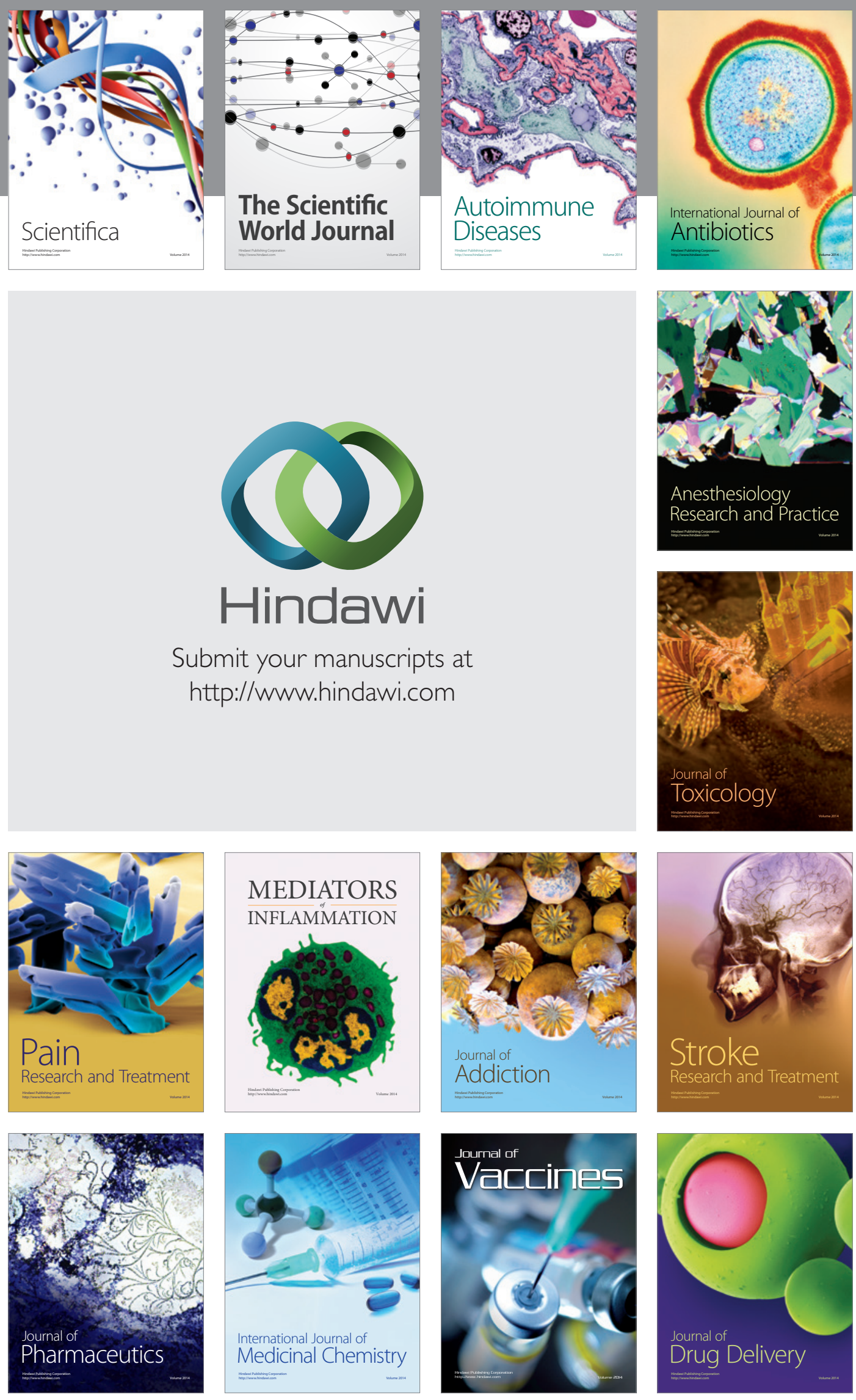